\title{
Prediction of concrete strength by non-destructive testing in old structures: Effect of core number on the reliability of prediction
}

\author{
Boussahoua Youcef $^{\text {a }}$, Kenai Said ${ }^{a}$ and Ali-Benyahia Khoudja ${ }^{\text {b }}$ \\ ${ }^{a}$ University of Blida1, Civil Engineering department, Blida, Algeria \\ ${ }^{\mathrm{b}}$ University of Khemis-Meliana, Civil Engineering department, Khemis-Meliana, Algeria
}

\begin{abstract}
When assessing the quality of concrete on site, it is necessary to base the interpretation on reliable and representative test results. Generally, core tests are the most reliable and effective method for assessing the quality of concrete. Unfortunately, this type of testing is expensive and time consuming and only a limited number of cores can be carried out in practice. Non-destructive tests (NDT) can be used to overcome these drawbacks. The most popular and widely used NDT methods for assessing concrete strength are rebound hammer (RH) and the ultrasonic pulse velocity (UPV). The use of these methods provide unreliable predictions unless their results are correlated to destructive tests. A sufficient number of cores is needed to accurately predict the compressive strength of concrete. Recent researches have been carried out for identifying and optimizing the number of cores able to stabilize the calibration model on data from recent structures and from synthetic data. However, more case studies are needed to draw conclusions. In addition, the effect of the degradation of reinforced concrete elements on the number of cores needed for obtaining a reliable prediction needs to be investigated. In this paper, RH and UPV in conjunction with core tests are used to evaluate the concrete compressive strength in existing structures built in the 1970s and degraded mainly by steel corrosion. More than 234 elements were tested by RH and 86 elements by UPV. Also, 36 cores were drilled and tested under compression. A regression analysis is adopted to establish the correlations between NDT and strength measurements. The accuracy of the predictive assessment was evaluated using two indicators: the root mean square error (RMSE) and the coefficient of determination $\left(\mathrm{r}^{2}\right)$. The results of this case study showed that seven to nine cores is the minimal number of cores that guarantees the improvement of concrete strength assessment by combined or single NDT methods.
\end{abstract}

Key words: non-destructive testing, concrete, cores, prediction, correlation, accuracy.

\section{Introduction}

Concrete is the most widely used materials worldwide in the construction industry due to its strength, possibility to realize all desired shapes and low cost. However, reinforced concrete structures may suffer significant deterioration that may threaten the stability of construction. There are many reasons for deterioration and degradation of concrete. Deterioration of concrete could be due to mechanical degradation, chemical attack, physical degradations or due to reinforcing steel corrosion. The latter is considered to be the most frequent anomaly encountered in reinforced concrete structures [1]. The compressive strength has a crucial role in the evaluation of concrete in existing buildings. Generally, core tests are the most reliable and effective method for assessing the quality of concrete in existing structures. Unfortunately, this type of testing is expensive and time consuming and also a limited number of cores can be carried out in practice.

In the field of civil engineering, non-destructive testing (NDT) are a valuable method for engineers and structure owners to establish precise diagnosis and to periodically monitor structures to ensure their safety without damaging them [2-4]. However, the use of NDT without cores test does not provide any reliable predictions of concrete strength as it is necessary to carry out a calibration operation to relate the concrete compressive strength of concrete and NDT measurements. In fact, it is difficult to establish this 
relationship [5], especially when dealing with old concrete structures [6] as the test results are dispersed and not easy correlated. For this reason, many researchers proposed various empirical models to relate RH and UPV measurements (separately or in combination) with the compressive strength [7-10]. The combined methods are known by SonReb methods. Some of these researchers performed their studies in laboratories and others on real structures. It is commonly agreed that none of these models can be used on all concretes and that it's necessary to establish models for each concrete with its own parameters. Core number is considered among the main keys for the accuracy and the reliability of assessment. The challenge on one hand is reducing the amount of core number without structurally affecting the concrete element or the structure and keeping the measurement at moderate costs and on the other hand to acquire a sufficiently complete and reliable understanding of the concrete strength in structures.

For this aim, recent research has been directed toward studying the effect of core number on the reliability of prediction. Authors in [11, 12] carried out an investigation using a synthetic simulation. More recently, Ali-Benyahia et al. [13] analysed these parameters on a recent real case study where 205 cores were tested and more than 155 structural elements (beams, columns and shear walls) were tested by RH and UPV. The main purpose of this study is to investigate the needed number of cores for a reliable strength assessment for old degraded structures.

\section{Case study presentation}

The case-study subject to the present investigation is a reinforced concrete industrial project composed of 17 different buildings built in the 70 s located in the north east of Algeria [14], classified according to the Algerian seismic regulations RPA99 / V2003 as a zone of average seismicity (zone IIa) [15]. The most important anomaly encountered is steel bars corrosion under the effect of carbonation as shown in Figure 1. This degradation is visible as spalling and delamination of concrete and cracks parallel to the steel bars.

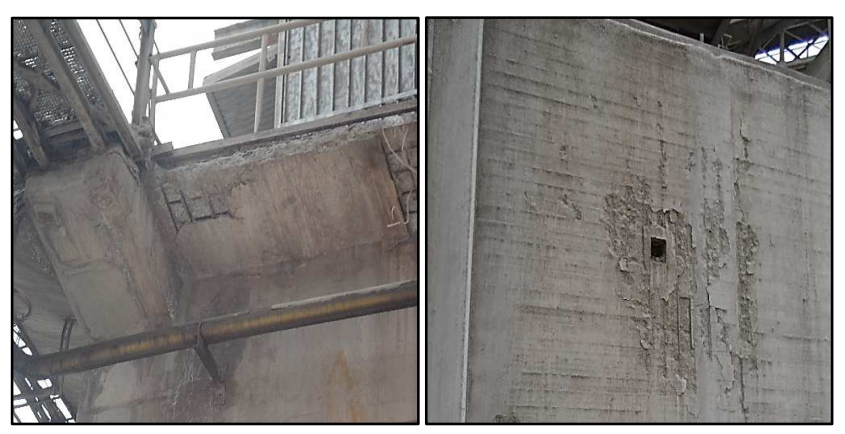

Figure 1: Typical degradation by steel corrosion

\section{Experimental program}

In order to clearly identify the quality of concrete in the structures, in-depth diagnosis was established. A detailed visual inspection was carried out to locate the anomalies and identify the elements to be tested. Then an NDT trial investigation was conducted. More than 230 rebound tests by Schmidt Hammer type N34 were carried out on three locations of each element with at least nine points per location. The UPV was measured by ultrasound in 86 elements by the direct method on at least three locations for each element after the preparation of the surfaces to be tested. Thirty-six (36) cores of $65 \mathrm{~mm}$ diameter were drilled and crushed in compression.

The cores were drilled in accordance with ASTM C42 [16]. The preparation of cores extremities and the tolerance of planarity and perpendicularity of the ends of the specimens are also prepared as described in NA 5071 [17].

In order to correct the influence of the ratio L/D (length to diameter) of the core on its compressive strength, and to convert the crushing core result to cylinder strength, the crushing results were multiplied by a correction coefficient (Table 1) according to ASTM C42 [16] if the ratio $\mathrm{L} / \mathrm{D} \leq 1.75$ as shown in the Table 1 :

Table 1: Coefficient of correction of core strength [16]

\begin{tabular}{cc}
\hline Ratio L/D & Correction Factor \\
\hline 1.75 & 0.98 \\
1.50 & 0.96 \\
1.25 & 0.93 \\
1.00 & 0.87 \\
\hline
\end{tabular}

\section{Statistical analysis}

The methodology adopted in this investigation is based on the comparison between two static parameters identifying the reliability of concrete strength prediction: the root mean square error (RMSE) Eq ( 1 ) and the coefficient of determination $\left(\mathrm{r}^{2}\right) \mathrm{Eq}(2)$ in order to analyse the effect of cores number on the prediction quality and also to identify the minimum number of cores sufficient to stabilise assessment models.

$$
\begin{aligned}
& \text { RMSE }=\sqrt{\sum_{i=1}^{n}\left(f_{c i}-\overline{f_{c l}}\right)^{2} / n} \\
& r^{2}=\frac{\sum_{i=1}^{n}\left(\overline{f_{c i}}-\overline{f_{c}}\right)^{2}}{\sum_{i=1}^{n}\left(f_{c i}-\overline{f_{c}}\right)^{2}}
\end{aligned}
$$

$f_{c i}=$ measured value; $\hat{f_{c r}}=$ estimated value; $\bar{f}_{\sigma}=$ arithmetic mean value. 
The flowchart presented in Figure 2 summarises the methodology adopted in this study. First, the algorithm is started by the data entry consisting of 36 triplets compromising from 36 crushing cores results and 36 results of the non-destructive tests (RH, UPV), the cores are drilled from the same location where NDT measurement were performed. Afterwards, a random selection was adopted by varying the number of core from 2 to 36 cores, and for each sample, 100 iterations was applied to minimize the error. Next, for each number of cores (NC) a calibration model was established, the rest of the results (N-NC) were used to test the quality of the estimation of the conversion model.

The regression analysis was adopted in our study, it is a statistical process for estimating the relationships among prediction variables and to establish a specific mathematic model. For modelling the relationship between the compressive strength and NDT measurements separately, one variable for the simple regression analysis was used. However, to establish the relationship between the compressive strength and $\mathrm{RH}$ and UPV measurements at the same time i.e two variables (combined method), a multiple regression analysis was performed.

Finally, for each approach, RMSE and $\mathrm{r}^{2}$ both were calculated as these parameters indicate the accuracy of the method. The variability was identified by the calculation of the standard deviation (Std Dev) and the results were verified with in-situ and compared to other methods used for strength assessment. This process is repeated (I) times with a step of one core.

\section{Results and discussion}

The accuracy of the estimation at the stages of calibration and prediction is presented as a function of number of cores on Figs. 3 to 5, respectively for RH and UPV. The quality of accuracy is tested by two statistical indicators RMSE and $r^{2}$. The results presented here are the mean values and their standards deviations of the indicators for the 100 simulations. For the low cores number, the calibration error is low whereas the prediction error is high (i.e the prediction capacity is low). When the cores number increases, the prediction capacity increases. Beyond nine (9) cores, the calibration and prediction errors (represented by RMSE and $\mathrm{r}^{2}$ ) stabilize about 6.5 $\mathrm{MPa}$ for RMSE and 0.22 for $\mathrm{r}^{2}$ for RH tests and 5.2 MPa (RMSE) and 0.50 (r2) for UPV.

Increasing furthermore the number of cores does not reduce the error in particular that of statistical origin.

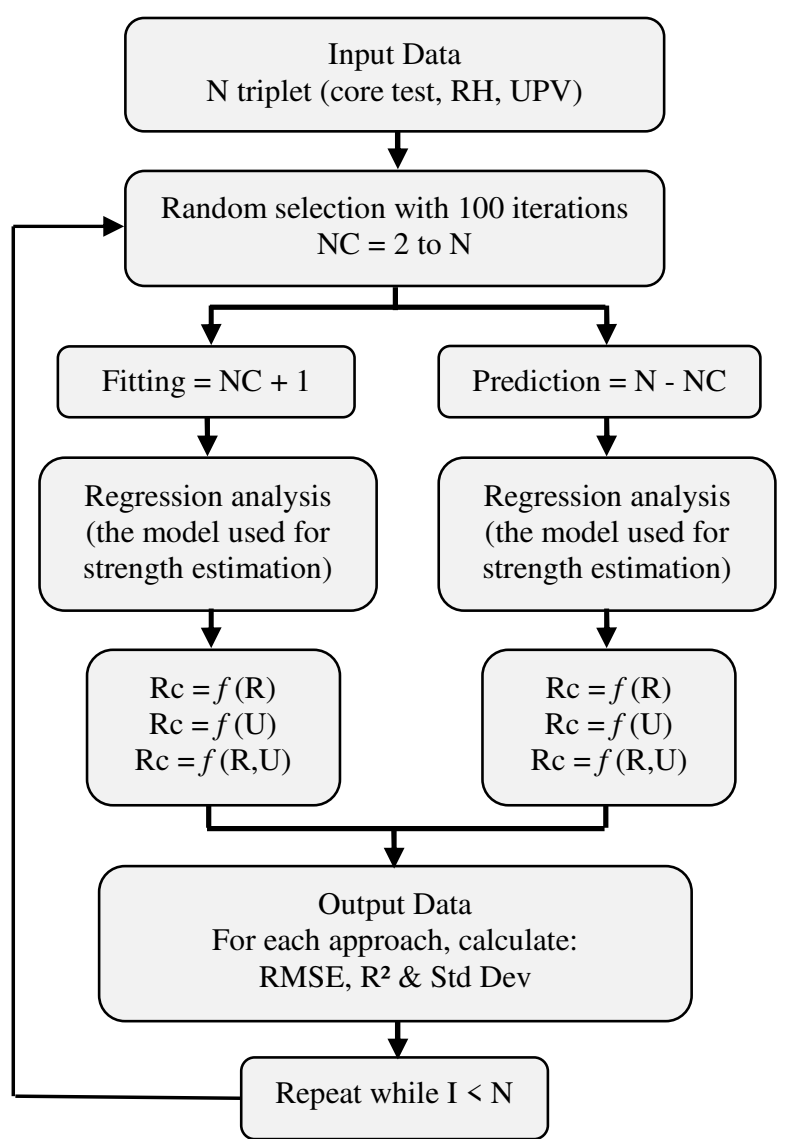

Figure 2: Flowchart represent the statistical analysis performed for strength assessment

These errors result from the variability of material, uncertainty in measurements, model errors, test location and the influence of uncontrolled parameters (humidity, carbonation, cracking, ..).

Alwash et al. [12] have tested the quality of accuracy of various models according to the number of cores using the statistical indicators RMSE for rebound and UPV. This study used synthetic data with three levels of measurement quality (low, medium and high). They concluded that beyond seven to eight cores the added value in terms of accuracy is low. In the case of RH, the error is stabilized at $4.5 \mathrm{MPa}, 3.5 \mathrm{MPa}$ or $2.5 \mathrm{MPa}$ for low, medium and high NDT measurement, respectively. For UPV, the error is stabilized respectively at 4.7 MPa, $3.8 \mathrm{MPa}$ and $2.8 \mathrm{MPa}$.

Similarly, Ali-Benyahia et al. [14] analyzed the effect of cores number on the quality of the accuracy of models based on data from a reel case study. The results showed that starting from nine cores, the error is stabilized at about $3 \mathrm{MPa}$ for RH and about $3.5 \mathrm{MPa}$ for UPV. 
According to these studies on synthetic data and reel cases studies (old and new constructions), it could be concluded that the number of cores affects considerably the error from statistical origin but for higher number of cores the effect is limited. In addition, the increase of cores number does not affect the non-statistical error (material variability, uncertainty in measurements and variability in uncontrolled parameters).
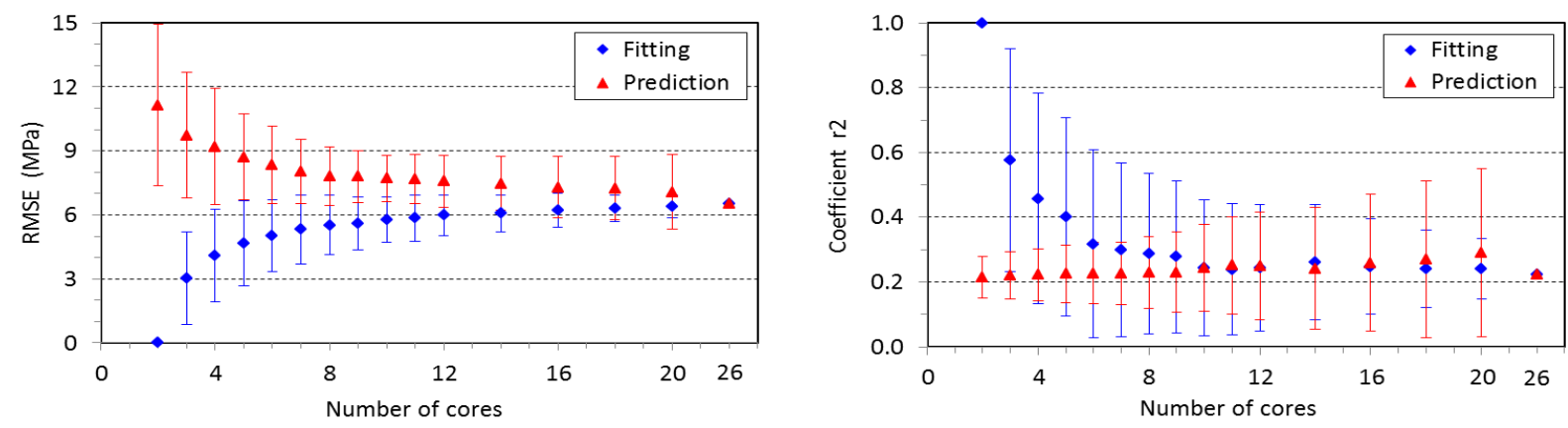

Figure 3: Effect of core number on the reliability of prediction using RH method
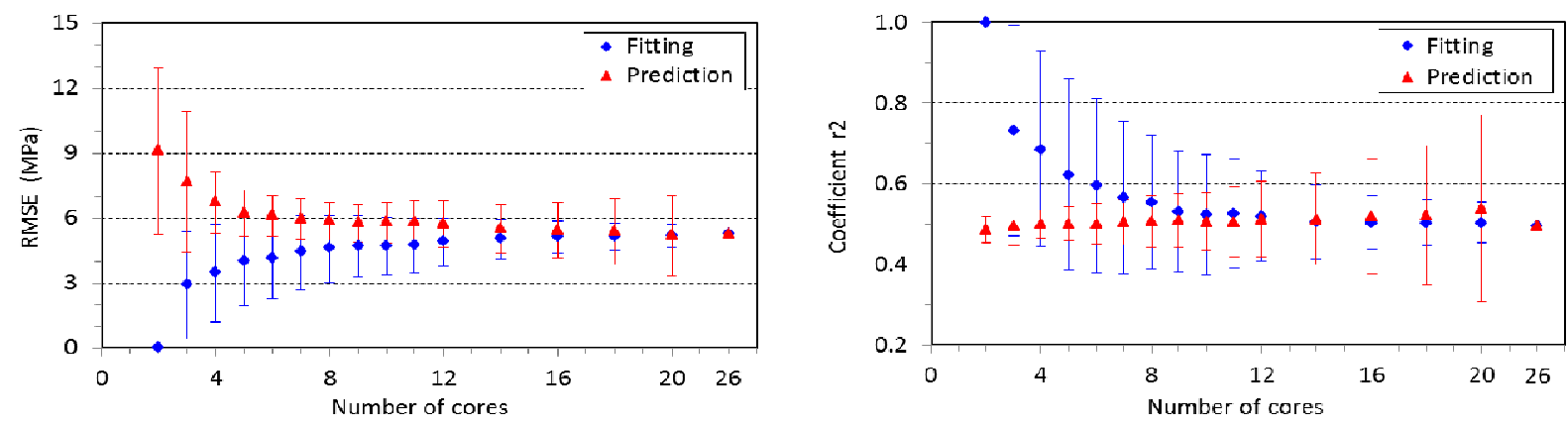

Figure 4: Effect of core number on the reliability of prediction using UPV method
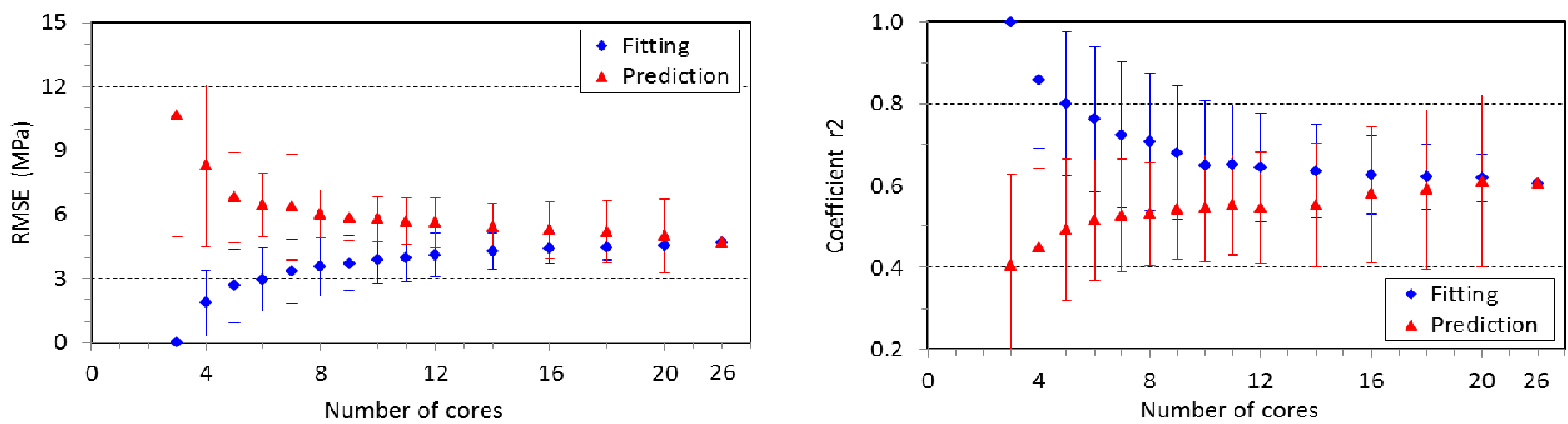

Figure 5: Effect of core number on the reliability of prediction using SonReb method.

\section{Conclusion}

A companion of destructive tests and NDT tests was carried out on exiting old reinforced concrete structures. A statistical analysis was performed to analyse the effect of concrete aging on NDT measurements and the reliability of strength assessment using two statistical indicators. From the results obtained for this case study, it can be concluded that:

- Seven to nine cores is the minimal number of cores that guarantees the improvement of concrete strength assessment by combined or single NDT methods. concrete on the stability and the reliability of the fit of regression models. 
Building Materials, vol. 33, pp. 139-63, (2012).

3. D. Breysse and O. Abraham, Méthodologie d'évaluation non-destructive de l'état d'altération des ouvrages en béton, France: Association Française de Génie Civil, (2005).

4. D. Breysse, Non-Destructive Assessment of Concrete Structures: Reliability and Limits of Single and Combined Techniques, RILEM, (2012).

5. S. Popovics, J. L. Rose and J. S. Popovics, "The behaviour of ultrasonic pulses in concrete," Cement and Concrete Research, vol. 20, pp. 259-270, (1990).

6. J. H. Bungey, S. G. Millard and M. G. Grantham, Testing of Concrete in Structures, London \& New York: Taylor \& Francis, (1992).

7. B. Hobbs and M. Tchoketch Kebir, "Nondestructive Testing Techniques for the Forensic Engineering Investigation of Reinforced Concrete Buildings," Forensic Science International, vol. 167, pp. 167-172, (2007).

8. H. Y. Qasrawi, "Concrete strength by combined nondestructive methods simply and reliably predicted," Cement and Concrete Research, vol. 30, pp. 739-746, (2000).

9. S. R. M. Khan, J. Noorzaei, M. R. A. Kadir, A. Waleed and M. S. Jaafar, "Effectiveness of sclerometer test technique on strength assessment of high performance concrete," International Journal of Engineering and Technology, vol. 1, no. 2, pp. 163168, (2004).

10. G. Kheder, "A two stage procedures for assessment of in situ concrete strength," Materials and Structures, vol. 32, pp. 410-417, (1999).

11. M. Alwash, D. Breysse and Z. M. Sbartaï, "Nondestructive strength evaluation of concrete: Analysis of some key factors using synthetic simulations," Construction and Building Materials, vol. 99, pp. 235-245, (2015).

12. M. Alwash, Z. M. Sbartaï and D. Breysse, "Nondestructive assessment of both mean strength and variability of concrete: A new bi-objective approach," Construction and Building Materials, vol. 113, pp. 880-889, (2016).

13. K. Ali-Benyahia, Z.-M. Sbartaï, D. Breysse, S. Kenai and M. Ghrici, "Analysis of the single and combined non-destructive test approaches for on-site concrete strength assessment: General statements based on a real case-study," CSCM Case Studies in Construction Materials, vol. 6, pp. 109-119, (2017).

14. S. Kenai and Y. Boussahoua, "Application des technique non destructifs pour l'inspection et diagnostic du béton armé dégradé" in Diagno-Béton, Merrakech, (2016).

15. DTR BC, "2 48. (2003). Règlement Parasismique Algérien -RPA 99 version 2003, CGS, Alger.
16. ASTM C 42/C 42M-04, Standard method of obtaining and testing drilled cores and sawed beams of concrete, Philadelphia, PA: American Society for Testing and Materials, (2001).

17. NA 5071, Tests for concrete in structures: Cores sampling, examination and testing compression, Algiers: Algerian Institute of Standardization, (2005). 